\title{
Aufkommens- und Verteilungsfolgen des Ersatzes des deutschen einkommensteuerlichen Formeltarifs durch einen Stufentarif
}

\section{Henriette Houben}

Leibniz Universität Hannover

Ralf Maiterth*

Humboldt-Universität zu Berlin

Heiko Müller

Ruhr-Universität Bochum

\section{Einleitung}

Seit Eintritt der FDP in die Bundesregierung im Herbst 2009 wird über die Einführung eines Stufentarifs anstelle des direkt-progressiven EinkommensteuerFormeltarifs, der zumindest in seiner Grundstruktur seit 1958 im Wesentlichen unverändert geblieben ist, diskutiert. Im Koalitionsvertrag der Regierungsparteien CDU, CSU und FDP ist vorgesehen, „Bezieher unterer und mittlerer Einkommen vorrangig $\mathrm{zu}$ entlasten und den Einkommensteuertarif $\mathrm{zu}$ einem Stufentarif umzubauen, der möglichst noch im Jahr 2011 in Kraft treten soll“ (CDU/CSU/FDP 2009, S. 10). Ein zentrales Argument zugunsten eines Stufentarifs ist die mit einer transparenten Tarifstruktur einhergehende Steuervereinfachung. Auch international sind Stufentarife gebräuchlich, wohingegen Formeltarife kaum anzutreffen sind. Ausgangspunkt der aktuellen politischen Diskussion in Deutschland war der erste von der FDP entwickelte Stufentarif, der drei Tarifstufen umfasste. Eine empirische Analyse der Aufkommens- und Arbeitsangebotswirkungen des FDP 3-Stufentarifs findet sich bei Neumann et al. (2009), welche zudem auch die Einführung eines Bürgergelds in ihre Analyse einbeziehen. Das RWI (2009) hat in seiner empirischen Analyse der Steuerprogramme zur Bundestagswahl 2009 u.a. die Be- und Entlastungswirkungen des FDP 3-Stufentarifs untersucht. Beide Untersuchungen belegen hohe Steuerausfälle, die mit der Einführung des FDP 3-Stufentarifs verbunden wären. Aufgrund dieser enormen Aufkommenseinbußen wurde der 3-Stufentarif schließlich verworfen, und es wurde ein 5-Stufentarif entwickelt, der lediglich Steuermindereinnahmen in der Größenordnung von rund 16 Mrd. $€$ bewirkt (FDP 2010, S. 7). Vor dem Hintergrund der Eurokrise und der auch in Deutschland notwendigen Konsolidierung der öffentlichen Haushalte

\footnotetext{
*Korrespondenzadresse: Prof. Dr. Ralf Maiterth, KPMG-Stiftungsprofessur für Betriebswirtschaftliche Steuerlehre, Humboldt-Universität zu Berlin, Dorotheenstr. 1, 10117 Berlin, E-Mail: ralf.maiterth@wiwi. hu-berlin.de.
} 


\section{Aufkommens- und Verteilungsfolgen des Ersatzes des deutschen einkommensteuerlichen Formeltarifs durch einen Stufentarif}

erscheinen Steuersenkungen in dieser Größenordnung derzeit nicht durchsetzbar. ${ }^{1}$ Dennoch ist nicht davon auszugehen, dass die FDP eines ihrer zentralen steuerpolitischen Ziele, die Einführung eines Stufentarifs, aufgibt. Vielmehr steht zu erwarten, dass eine mehr oder minder aufkommensneutrale Tarifreform in Angriff genommen wird. Aus diesem Grunde wurden im Rahmen des vorliegenden Beitrags einige Stufentarife entwickelt, die nahezu aufkommensneutral wirken. Bei der Entwicklung dieser Stufentarife spielten neben den Aufkommenseffekten auch Verteilungswirkungen und der Aspekt der Einfachheit im Sinne von Tariftransparenz eine wichtige Rolle.

Der vorliegende Beitrag analysiert die Aufkommens- und Verteilungswirkungen der Substitution des Einkommensteuer-Formeltarifs durch einen Stufentarif empirisch auf Grundlage der fortgeschriebenen Mikrodaten der amtlichen Einkommensteuerstatistik 2004. Ausgangspunkt der vorliegenden Untersuchung ist der FDP 3-Stufentarif. Darüber hinaus wird der FDP 5-Stufentarif untersucht, wenngleich der Schwerpunkt des Beitrags auf der Analyse der Aufkommensund Verteilungswirkungen von zum Einkommensteuertarif 2010 alternativen und nahezu aufkommensneutralen Stufentarifen liegt. Zudem wird diskutiert, ob die betrachteten Stufentarife auch dem Kriterium der Transparenz genügen. Vorgeschaltet ist eine Analyse der Änderung des Einkommensteuer-Formeltarifs im Jahr 2010, die es dem Leser erleichtern soll, die Aufkommens- und Verteilungswirkungen der Einführung eines Stufentarifs zu werten.

\section{Modell und Datengrundlage}

\subsection{Datengrundlage und Datenfortschreibung}

Als Datengrundlage für den vorliegenden Beitrag dient die Faktisch Anonymisierte Lohn- und Einkommensteuerstatistik aus dem Jahre 2004 (FAST 2004), die das Statistische Bundesamt der Wissenschaft in Form eines Scientific Use File zur Verfügung stellt. $^{2}$ Es handelt sich um echte Veranlagungsdaten. FAST 2004 basiert auf der 10\%-Stichprobe i.S.d. § 7 Abs. 4 StStatG der Lohnund Einkommensteuerstatistik 2004. Es handelt sich um eine geschichtete Zufallsstichprobe. $^{3}$ Die darin enthaltenen Datensätze weisen knapp 1.000 für die Einkommensteuerveranlagung relevante Merkmale von ca. 3,5 Mio. Steuerpflichtigen aus, die hochgerechnet ca. 35 Mio. Steuerpflichtige und - da im Fall zusammenveranlagter Eheleute ein Steuerpflichtiger zwei Personen umfasst insgesamt rund 51 Mio. Personen repräsentieren. Die verwendete Datenbasis gewährleistet somit repräsentative Simulationsergebnisse.

Aufgrund der mannigfaltigen Probleme einer exakten Datenfortschreibung ${ }^{4}$ wurde lediglich die einkommensteuerliche Bemessungsgrundlage - ohne Berücksichtigung sozio-demographischer Veränderungen _ einheitlich

1. Der Sachverständigenrat (2009), S. 11, hält sogar Steuererhöhungen für nahezu unumgänglich.

2. Vgl. ausführlich zu FAST Kordsmeyer (2004).

3. Zu Einzelheiten der Stichprobenziehung im Rahmen der amtlichen Einkommensteuerstatistik vgl. Zwick und Meyer (1998), S. 570 -573.

4. Hier sei beispielsweise auf die Probleme bei einer Fortschreibung der von den Steuerpflichtigen erzielten Einkommen hingewiesen, bei der sich insbesondere die Prognose der Entwicklung steuerlicher Verluste schwierig gestaltet (vgl. zur Entwicklung einkommensteuerlicher Verluste Müller 2007). 
entsprechend den Zuwachsraten der volkswirtschaftlichen Referenzgröße „Volkseinkommen“ fortgeschrieben. ${ }^{5}$ Dabei wurde von einem Nominalwachstum des Volkseinkommens von 2004 bis einschließlich 2008 in Höhe von rund 12,78\% ausgegangen. ${ }^{6}$ Aufgrund des Rückgangs des Volkseinkommens in den ersten beiden Quartalen 2009 und dem folgenden Anstieg in den letzten beiden Quartalen 2009 sowie der unsicheren wirtschaftlichen Entwicklung dürfte das Einkommensniveau des Jahres 2008 eine gute Approximation für das Einkommen 2010 darstellen.

\subsection{Mikrosimulationsmodell}

Die vorliegende Untersuchung basiert auf einem statischen Mikrosimulationsmodell, welches die Auswirkungen der Besteuerung auf die Primäreinkommensverteilung aufgrund steuerbedingter Verhaltensänderungen nicht berücksichtigt. ${ }^{7}$ Das Augenmerk liegt auf der Verteilung der Steuerzahlungen auf die Wirtschaftssubjekte; es handelt sich folglich um eine formale Inzidenzanalyse. ${ }^{8}$

Für diese Untersuchung wird das Einkommen als Summe aus dem zu versteuernden Einkommen, den abgeltungsbesteuerten Einkünften aus Kapitalvermögen und den Einkünften, die dem Progressionsvorbehalt des $\S 32 \mathrm{~b}$ EStG unterliegen, definiert. Da sich die verteilungspolitischen Implikationen kaum unterscheiden, wird in der Darstellung und Analyse der Ergebnisse auf eine Differenzierung zwischen Einzel- und Zusammenveranlagten verzichtet. Zur personenbezogenen Auswertung erfolgte eine Umrechnung des steuerlichen Einkommens sowie der Einkommensteuer auf Größen je Kopf. D.h., zusammenveranlagte Eheleute werden als zwei Personen mit jeweils identischem steuerlichen Einkommen und identischer Steuerlast behandelt. ${ }^{9}$

Für die Berechnung der Einkommensteuer wird in der vorliegenden Untersuchung auf die tarifliche Einkommensteuer abgestellt. ${ }^{10}$ Dabei finden neben dem allgemeinen Einkommensteuertarif gemäß $\S 32 \mathrm{a}$ EStG auch

5. Bei der Datenfortschreibung wurde eine gleichmäßige Entwicklung bei sämtlichen Einkunftsarten ebenso unterstellt wie eine unveränderte Einkommensverteilung. Die nachfolgenden Ergebnisse basieren folglich auf der Einkommensverteilung des Jahres 2004.

6. Vgl. http://www.destatis.de/jetspeed/portal/cms/Sites/destatis/Internet/DE/Content/Statistiken/ VolkswirtschaftlicheGesamtrechnungen/Inlandsprodukt/Tabellen/Volkseinkommen1950.psml.

7. Für die vorliegende Untersuchung wird ein statisches Mikrosimulationsmodells verwendet, da u.E. für Deutschland keine eindeutigen empirischen Erkenntnisse über die Verhaltensreaktionen der Steuerpflichtigen auf eine Tarifänderung vorliegen. Es ist national wie international durchaus üblich, statische Mikrosimulationsmodelle zu verwenden (vgl. für Deutschland bspw. Bork 2002, Merz und Zwick 2002, S. 729, van Essen, Kaiser und Spahn 1988, RWI 2009, als Beispiele für internationale statische Mikrosimulationsmodelle seien EUROMOD - dabei handelt es sich um ein „Tax-BenefitModell“ für fünfzehn europäische Länder - und STINMOD - ein Mikrosimulationsmodell für Australien - genannt (vgl. zu EUROMOD Sutherland 2000 und zu STINMOD Lambert et.al. 1994)).

8. Eine derartige Analyse haben z.B. van Essen, Kaiser und Spahn zur Beurteilung der Verteilungswirkungen der Einkommensteuerreformen 1986 - 1990 vorgenommen (vgl. van Essen, Kaiser und Spahn 1988).

9. Auf eine Verwendung von Äquivalenzeinkommen auf Haushaltsebene wurde verzichtet, da es nicht möglich ist, die Personen Haushalten zuzuordnen. So ist bspw. bei allein veranlagten Steuerpflichtigen unbekannt, mit welchem Steuerpflichtigen sie ggf. in einem Haushalt leben.

10. Hinzurechnungen und Kürzungen, die für die Berechnung der festzusetzenden Einkommensteuer zu berücksichtigen sind, wie bspw. die Anrechnung ausländischer Steuern oder die Hinzurechnung des Kindergeldes bei Inanspruchnahme des Kinderfreibetrags, werden daher nicht berücksichtigt. 


\section{Aufkommens- und Verteilungsfolgen des Ersatzes des deutschen einkommensteuerlichen Formeltarifs durch einen Stufentarif}

der Progressionsvorbehalt gemäß $\S 32 b$ EStG, die Abgeltungsteuer gemäß $\S 32 \mathrm{~d}$ EStG sowie die Besteuerung der außerordentlichen Einkünfte gemäß $\S 34$ EStG Berücksichtigung. Die Besteuerung gemäß $\S 34 \mathrm{a}$ EStG wird in den Simulationsrechnungen nicht berücksichtigt, da diese Vorschrift nur in wenigen Ausnahmefällen eine Begünstigung darstellt ${ }^{11}$ und keine empirischen Daten zur tatsächlichen Inanspruchnahme der Vorschrift vorliegen. Auch der Solidaritätszuschlag bleibt unberücksichtigt, da der Solidaritätszuschlag bei der Konzeption von Einkommensteuertarifen zumindest im politischen Prozess keine Rolle spielt. Dies zeigt sich besonders bei der FDP, die in ihrem auf dem 59. Bundesparteitag verabschiedeten Steuerkonzept das Auslaufen des Solidaritätszuschlags im Jahr 2019 beschlossen hat (FDP 2008, S. 8). Bei der Berechnung der Steuerlast für Kapitaleinkünfte wird unterstellt, dass der Steuerpflichtige die Veranlagungsoption gemäß $\S 32 \mathrm{~d}$ Abs. 6 EStG immer dann ausübt, wenn die Abgeltungsteuer höher als die Steuer ist, die sich ergeben würde, wenn die Einkünfte aus Kapitalvermögen gemäß $\S 32 \mathrm{a}$ EStG besteuert würden.

Durch die Berücksichtigung der verschiedenen Tarifvorschriften kann sich die für zwei Steuerpflichtige mit identischem Einkommen in den Simulationsrechnungen ergebende Einkommensteuer unterscheiden, je nachdem mit welchem Anteil abgeltungsbesteuerte und außerordentliche Einkünfte im Einkommen enthalten sind. Somit ist es auch möglich, dass bei identischen Einkommen ein Steuerpflichtiger von einer Tarifänderung profitiert, wogegen ein anderer Steuerpflichtiger mit gleich hohem Einkommen $\mathrm{zu}$ den Verlierern gehört. Um trotzdem empirisch einkommensabhängige Gewinner-/Verlierer-Bereiche identifizieren und ausweisen $\mathrm{zu}$ können, wurden die Simulationsergebnisse in halben Perzentil-Schritten klassiert. Es wird somit in der Analyse darauf abgestellt, wie sich eine Tarifänderung bspw. auf die 0,5\% der Personen mit dem geringsten Einkommen im Durchschnitt auswirkt. Die im Folgenden angegebenen Einkommensbereiche der empirisch ermittelten Gewinner- und Verlierergruppen basieren jeweils auf den Grenzen der halben Perzentile.

\section{Aufkommens- und Verteilungswirkungen der Tarifänderung 2010}

Die Änderung des Formeltarifs gemäß $\S 32 \mathrm{a}$ EStG im Jahr 2010 besteht in der moderaten Erhöhung der Tarifzonengrenzen, wie Tabelle 1 zeigt.

Der Tarif 2010 verursacht im Vergleich mit dem Tarif 2009 Steuermindereinnahmen i.H.v. 2,2 Mrd. $€{ }^{12}$ Der empirisch ermittelte, aggregierte Durchschnittssteuersatz (Steuerquote) ${ }^{13}$ sinkt von $20,02 \%$ auf $19,80 \%$.

11. Siehe Homburg, Houben und Maiterth (2008) und Houben und Maiterth (2008).

12. Das RWI errechnet auf Basis der Daten des Scientific Use Files der Einkommensteuerstatistik 2001 (FAST 2001) Steuermindereinnahmen infolge der Tarifänderung 2010 i.H.v. 2,25 Mrd. € (vgl. RWI 2009, S. 26). Boss et al. (2009) ermitteln einen Steuerausfall des Tarifs 2010 gegenüber dem 2009er Tarif im Hinblick auf das Lohnsteueraufkommen i.H.v. 2,01 Mrd. € (vgl. Boss et al. 2009, S. 5).

13. Der aggregierte Durchschnittssteuersatz ist der Quotient aus dem Steueraufkommen und der Summe aus dem aggregierten zu versteuernden Einkommen und den aggregierten abgeltungsbesteuerten Kapitaleinkünften. 
Tabelle 1 Tarifzonengrenzen und ausgewählte Grenzsteuersätze der Einkommensteuertarife 2009 und 2010.

\begin{tabular}{lccc}
\hline & 2009 & Änderung & \multicolumn{1}{c}{2010} \\
\hline $\begin{array}{l}\text { Beginn der ersten } \\
\text { Progressionszone }\end{array}$ & $7.835 €(14 \%)$ & $170 €$ & $8.005 €(14 \%)$ \\
$\begin{array}{l}\text { Intervallgrenze innerhalb der } \\
\text { Progressionszone }\end{array}$ & $13.140 €(24 \%)$ & $330 €$ & $13.470 €(24 \%)$ \\
$\begin{array}{l}\text { Beginn obere } \\
\text { Proportionalzone } \\
\text { Beginn der } \\
\text { "Reichensteuer" }\end{array}$ & $52.552 €(42 \%)$ & $330 €$ & $52.882 €(42 \%)$ \\
\hline
\end{tabular}

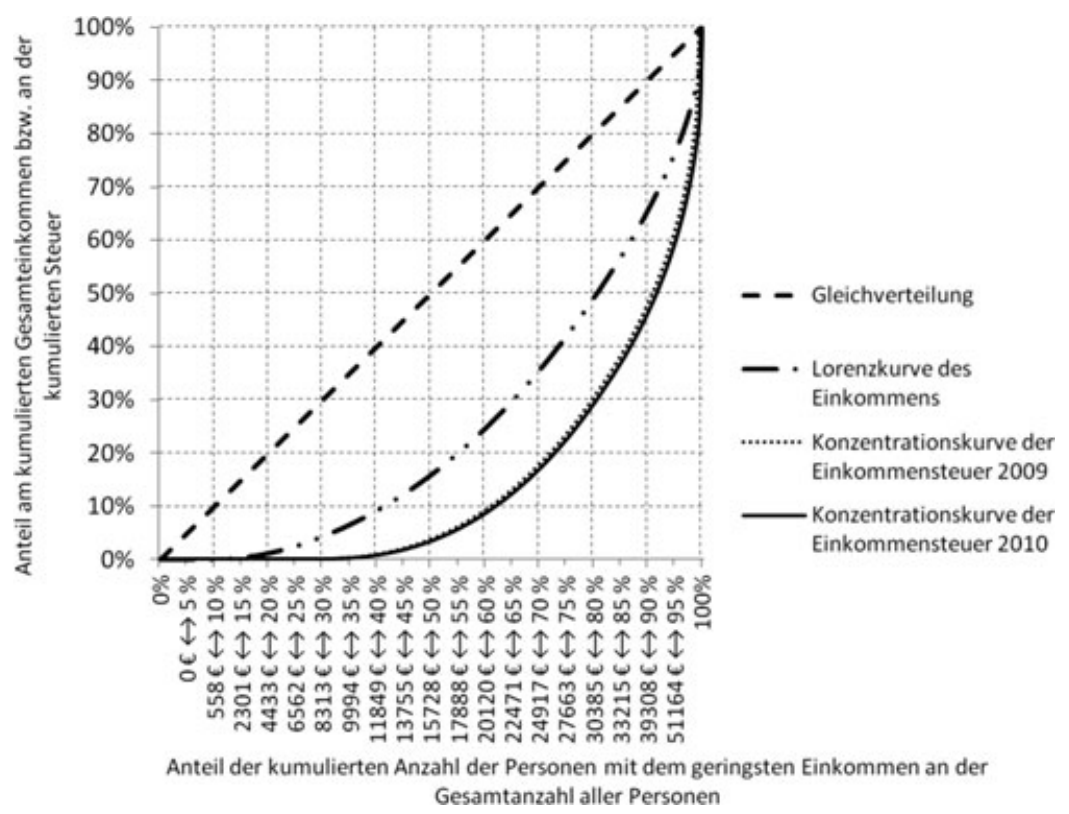

Abbildung 1 Lorenzkurve des steuerlichen Einkommens und Konzentrationskurve der Einkommensteuerlast 2009 und 2010 pro Person.

Die in Abbildung 1 dargestellte Lorenzkurve ${ }^{14}$ des steuerlichen Einkommens und die Konzentrationskurven ${ }^{15}$ der tariflichen Einkommensteuer 2009 und 2010 zeigen die empirische Verteilung des Einkommens und der korrespondierenden Steuerbelastung.

Wie die Darstellung der empirischen Einkommensverteilung in Abbildung 1 erkennen lässt, weist die Hälfte aller Personen ein steuerliches Einkommen

14. Dabei sind auf der $\mathrm{x}$-Achse der Anteil der Steuerpflichtigen sortiert nach ihrem Einkommen abgetragen. Die y-Achse gibt den auf diese Bevölkerungsgruppe entfallenden Anteil des kumulierten steuerlichen Einkommens wieder.

15. Dabei sind auf der $\mathrm{x}$-Achse der Anteil der Steuerpflichtigen sortiert nach ihrem Einkommen abgetragen. Die y-Achse gibt den auf diese Bevölkerungsgruppe entfallenden Anteil der kumulierten Steuerlast wieder. Die Konzentrationskurve unterscheidet sich von einer Lorenzkurve darin, dass die Personen nicht nach dem auf der y-Achse abgetragenen Merkmal sortiert sind. 


\section{Aufkommens- und Verteilungsfolgen des Ersatzes des deutschen einkommensteuerlichen Formeltarifs durch einen Stufentarif}

Tabelle 2 Konzentrationsindex, Gini-Koeffizient und Kakwani-Progressionsmaß für den Einkommensteuertarif 2009 und 2010.

\begin{tabular}{lccr}
\hline & 2009 & Änderung & 2010 \\
\hline Konzentrationsindex der Steuer & 0,712 & $0,4 \%$ & 0,715 \\
Gini-Koeffizient des Einkommens & 0,503 & - & 0,503 \\
Kakwani-Progressionsmaß & 0,209 & $1,5 \%$ & 0,212 \\
\hline
\end{tabular}

unter $15.729 €$ (Medianeinkommen) auf. Ein Viertel verfügt sogar nur über ein Pro-Kopf-Einkommen von weniger als $6.562 €$ und $7,6 \%$ aller Personen über ein steuerliches Einkommen kleiner gleich null. $\mathrm{Zu}$ den 10\% einkommensstärksten Personen gehört, wessen steuerliches Einkommen $39.308 €$ übersteigt.

Abbildung 1 zeigt zudem, dass in Deutschland aufgrund des progressiven Tarifs die Einkommensteuerbelastung erheblich ungleicher verteilt ist als das Einkommen. Unter Anwendung des Tarifs 2009 zahlen rund 29\% aller Personen überhaupt keine Einkommensteuer; unter Anwendung des Tarifs 2010 etwa 29,5\%. Diejenigen 50\% der Einkommensbezieher, deren Einkommen unterhalb des Medianeinkommens liegt und die knapp 16\% des aggregierten Einkommens auf sich vereinigen, tragen beim Tarif 2009 lediglich 3,6\% und beim Tarif 2010 nur 3,4\% zum Einkommensteueraufkommen bei. Dagegen entfällt auf die Personen im obersten Einkommensdezil 53,2\% der Einkommensteuer 2009 bzw. 53,5\% der Einkommensteuer 2010, obwohl sie nur 34,5\% des aggregierten Einkommens erwirtschaften. Die Einkommensstärksten 1\% erzielen 10,9\% des Einkommens und tragen 2009 einen Anteil i.H.v. 20,9\% und 2010 i.H.v. 21,1\% der Einkommensteuerlast.

Die im Vergleich zum Einkommen erheblich ungleichere Verteilung der Einkommensteuerlast belegt auch der Vergleich zwischen dem Gini-Koeffizient als globales Maß für die Verteilung des Einkommens und dem Konzentrationsindex als globales Maß für die Verteilung des Einkommensteuer in Tabelle 2. ${ }^{16}$ Darüber hinaus ist in Tabelle 2 auch das Kakwani-Progressionsmaß ausgewiesen, das angibt, um wie viel ungleicher die Steuer im Vergleich zum Einkommen verteilt ist (Kakwani 1977).

Die Ergebnisse in Tabelle 2 zeigen, dass die Ungleichheit der Steuerlastverteilung im Vergleich der Tarife 2009 und 2010 moderat steigt.

Anhand der globalen Verteilungsmaße aus Tabelle 2 lassen sich lediglich Aussagen über Verteilungswirkungen von Tarifänderungen in ihrer Gesamtheit treffen. Es kann jedoch nicht abgeleitet werden, wie sich eine Tarifänderung auf einzelne Gruppen in Anhängigkeit von deren Einkommen auswirkt. Als Maßstab zur Bestimmung von Gewinnern und Verlierern von Tarifänderungen stellt der vorliegende Beitrag auf die Veränderung der Steuerlastquote bzw. des Durchschnittssteuersatzes ab. Folglich sind Gewinner einer Tarifänderung

16. Die Fläche zwischen der Lorenzkurve und der 45-Grad-Kurve stellt eine Maßgröße dafür dar, wie ungleich das Einkommen verteilt ist. Das Doppelte dieser Fläche wird als Gini-Koeffizient bezeichnet. Verdoppelt man die Fläche zwischen der Konzentrationskurve und der 45-Grad-Linie, so erhält man den Konzentrationsindex. 
Henriette Houben, Ralf Maiterth und Heiko Müller

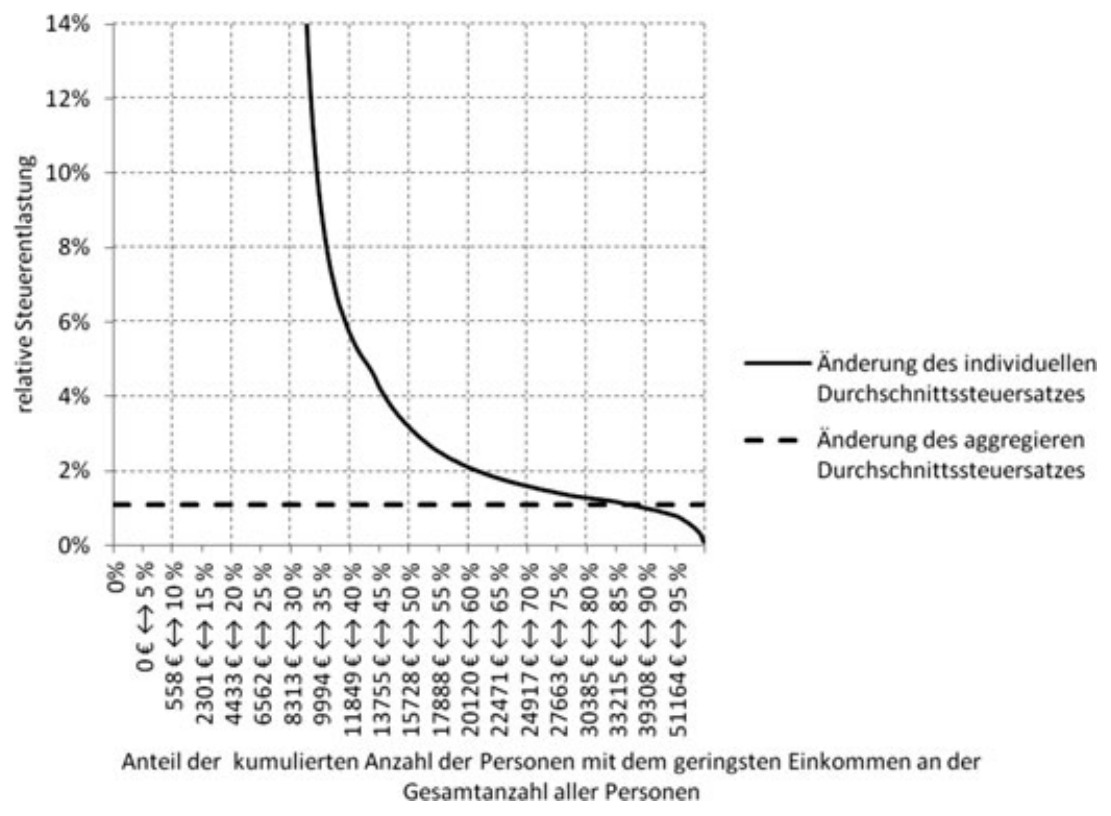

Abbildung 2 Gewinner und Verlierer der Tarifänderungen 2010.

Personen, deren individuelle Durchschnittssteuerbelastung stärker sinkt bzw. geringer steigt als der aggregierte Durchschnittssteuersatz. ${ }^{17}$

Abbildung 2 veranschaulicht die Gruppen der Gewinner und Verlierer der Tarifänderung 2010 grafisch, wobei positive Werte auf der Ordinate eine Reduktion des Durchschnittssteuersatzes und negative Werte eine Erhöhung des Durchschnittssteuersatzes bedeuten.

Wie bereits erwähnt, sinkt im Vergleich der Tarife 2009 und 2010 der empirisch ermittelte aggregierte Durchschnittssteuersatz von 20,02\% um 0,22 Prozentpunkte auf 19,80\%, was einer durchschnittlichen Entlastung i.H.v. 1,09\% entspricht. Im Einkommensbereich zwischen $7.665 €$ und dem Grundfreibetrag des Tarifs 2010 i.H.v. 8.004 $€$ beläuft sich die Entlastung auf 100\%, da unter Anwendung des Tarifs 2010 nunmehr keine Einkommensteuer zu zahlen ist. Diese Personen erfahren die höchste relative Entlastung. Mit zunehmendem Einkommen nimmt die relative Entlastung ab. 29\% aller Personen - dies sind 14,68 Mio. - werden von der Tarifänderung nicht betroffen, da sie bereits 2009 keine Einkommensteuer zahlten. Rund 58\% derjenigen Personen, die 2009 mit Einkommensteuer belastet waren, profitieren von der Tarifänderung 2010 überproportional; dies sind gut 29,37 Mio. Personen. Diese Personen verfügen über ein Einkommen zwischen dem Grundfreibetrag und $35.287 €$. Die 13\% der Personen mit den höchsten Einkommen (Einkommen über $35.287 €$ ) - dies sind knapp 6,58 Mio.

17. Umverteilungsneutralität ist damit im hier verstandenen Sinne gegeben, wenn die Steuerentlastung in Bezug zur Steuerschuld vor der Tarifänderung (relative Steuerentlastung) bei allen Steuerpflichtigen gleich hoch ist. In dieser Diktion gilt eine Tarifänderung als umverteilungsneutral, wenn der Anteil der einzelnen Steuerpflichtigen am Gesamtsteueraufkommen unverändert bleibt. Dies ist äquivalent zu einer einheitlichen Änderung des Durchschnittssteuersatzes sämtlicher Steuerpflichtigen. Vgl. dazu ausführlich Maiterth und Müller (2009). 


\section{Aufkommens- und Verteilungsfolgen des Ersatzes des deutschen einkommensteuerlichen Formeltarifs durch einen Stufentarif}

Personen - zählen dagegen zu den Verlierern der Tarifänderung, da ihre relative Entlastung unter der durchschnittlichen Entlastung liegt. Beispielsweise beträgt für einen einzelveranlagten Steuerpflichtigen mit einem ausschließlich nach $\S 32 \mathrm{a}$ EStG zu versteuernden Einkommen i.H.v. $200.000 €$ die relative Entlastung nur 0,34\%.

\section{Aufkommens- und Verteilungswirkungen der FDP-Stufen-Tarife}

\subsection{Aufkommens- und Verteilungswirkungen des FDP-3-Stufentarifs}

Der im Beschluss des 59. Bundesparteitages vom Juni 2008 von der FDP geforderte Tarif sieht - wie der Einkommensteuertarif 2010 - einen Grundfreibetrag in Höhe von $8.004 €$ vor, hat drei Tarifstufen und damit drei verschiedene Grenzsteuersätze i.H.v. 10\%, 25\% und 35\%. Die Tarifstufenobergrenzen weisen mit $20.000 €$ und $50.000 €$ runde Beträge auf, die offensichtlich der Einfachheit und Transparenz dienen sollen. ${ }^{18}$ In Abbildung 3 sind sowohl die Grenz- als auch die Durchschnittssteuersätze des Einkommensteuertarifs 2010 und des FDP-3Stufentarifs dargestellt.

Der FDP-3-Stufentarif weist gegenüber dem Formeltarif 2010 erheblich niedrigere Grenzsteuersätze aus, von denen sich die FDP u.a. Wachstumsimpulse verspricht. $^{19}$ Die deutlich niedrigeren Durchschnittssteuersätze führen zu beträchtlichen Steuermindereinnahmen. Die im Vergleich zum Formeltarif 2010 berechneten Steuermindereinnahmen betragen 63,59 Mrd. €; dies entspricht einem Steuerausfall i.H.v. 31,91\% des Einkommensteueraufkommens. Der aggregierte Durchschnittssteuersatz sinkt von 19,80\% um beachtliche 6,32 Prozentpunkte bzw. $31,91 \%$ auf $13,48 \% .^{20}$

18. Neben der Einführung eines 3-Stufentarifs sieht das Steuerkonzept der FDP zahlreiche Änderungen der Steuerbemessungsgrundlage vor. Diese reichen von einer Werbungskostenpauschale für Arbeitnehmer i.H.v. 2\% der Einnahmen, mindestens jedoch $200 €$ und höchstens $5000 €$, über die vollständige Abzugsfähigkeit von Beiträgen zur sozialen Sicherung bis zur Gewährung des Grundfreibetrags auch für Kinder (inkl. Anhebung des Kindergelds auf $200 €$ pro Kind). Weiterhin ist die Abschaffung der Abgeltungsteuer auf Veräußerungsgewinne geplant. Der Solidaritätszuschlag soll, wie bereits erwähnt, 2019 auslaufen. Kinderbetreuungs- und Pflegekosten sollen bis 12.000 $€$ vollständig abziehbar sein. Darüber hinaus ist eine Begrenzung des Einkommensteuersatzes bei unternehmerischen Einkünften auf 28\% vorgesehen und Dividenden sollen im Ausschüttungsfall nicht zusätzlich mit Einkommensteuer belastet werden. Im Bereich der Unternehmensbesteuerung sollen die Zinsschranke, die Mindestbesteuerung und die Einschränkungen der Verlustnutzung in Übernahmefällen abgeschafft werden. Schließlich soll die degressive Abschreibung wieder eingeführt und die Sofortabschreibung für geringwertige Wirtschaftsgüter bis $1.000 €$ zugelassen werden. Die Wirkungen der vorgeschlagenen Änderungen der Bemessungsgrundlage sind aufgrund der Datenlage nicht mit einer der Tarifsimulation vergleichbaren Präzision quantifizierbar. Es ist jedoch zu vermuten, dass die beabsichtigten Änderungen zusätzliche Steuermindereinnahmen in nicht unerheblichem Umfang hervorrufen. Eine explizite Berücksichtigung der vorgeschlagenen Änderungen der Bemessungsgrundlage bleibt im vorliegenden Beitrag jedoch insbesondere deshalb unberücksichtigt, um die reinen Tarifwirkungen herauszuarbeiten. Andernfalls ließen sich die gewonnenen Ergebnisse nicht mit denen der weiteren im Beitrag betrachteten Tarife vergleichen.

19. Die Ergebnisse des vorliegenden Beitrags wurden, wie bereits erwähnt, unter Vernachlässigung von Wachstumseffekten ermittelt. Damit handelt es sich bei den hier präsentierten Ergebnissen um first-round-Effekte. Simulationsrechnungen zur Beschäftigungswirkung des FDP-Vorschlags haben Neumann, Peichl, Schneider und Siegloch (2009) durchgeführt.

20. Neumann, Peichl, Schneider und Siegloch berechnen mittels des Simulationsmodells des IZA auf Basis der 2007er Welle des Sozioökonomischen Panels (SOEP) eine Aufkommensminderung i.H.v. 


\section{Henriette Houben, Ralf Maiterth und Heiko Müller}

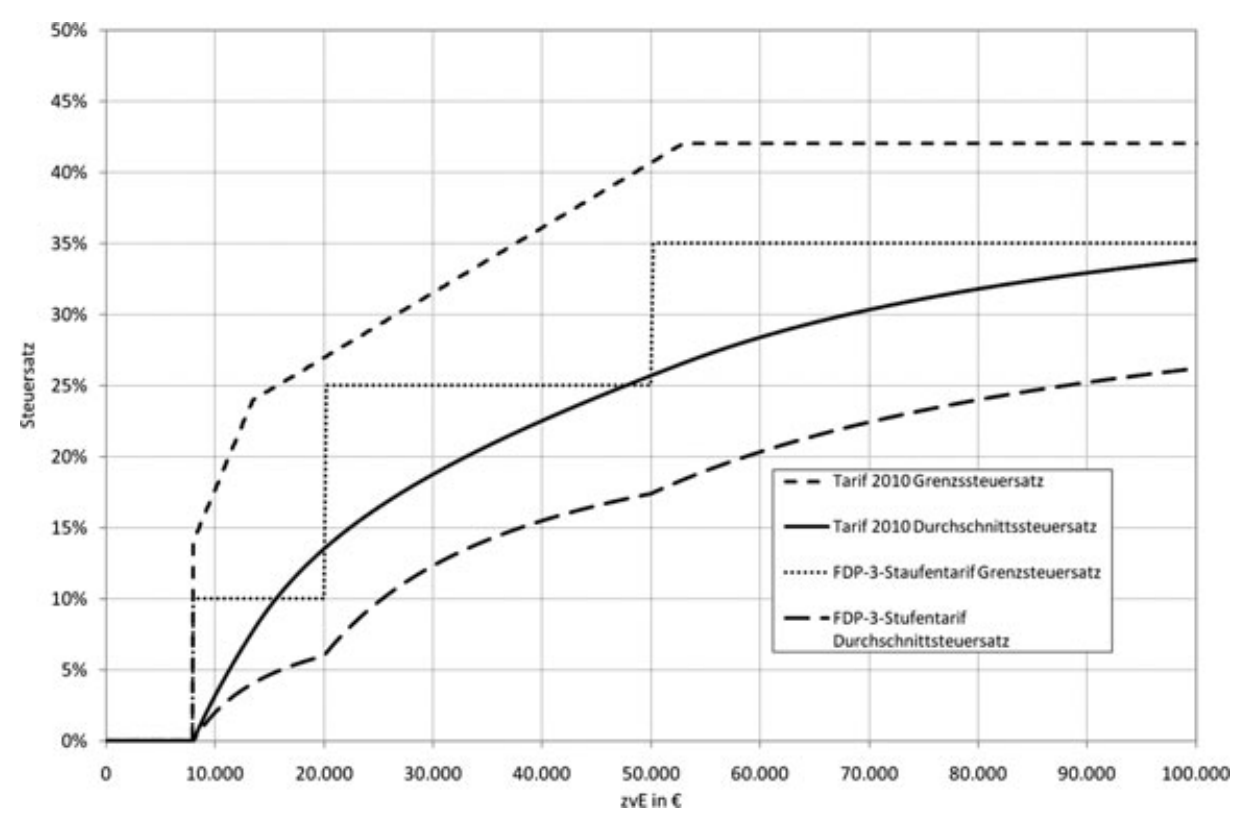

Abbildung 3 Grenz- und Durchschnittssteuersatz beim Formeltarif 2010 und FDP-3-Stufentarif.

Von besonderem Interesse sind die Verteilungswirkungen des FDP-3Stufentarifs, da die FDP in weiten Teilen der Bevölkerung als Klientelpartei der „Besserverdienenden“ wahrgenommen wird. Aus diesem Grunde liegt a priori die Vermutung nahe, der FDP-3-Stufentarif würde Bezieher hoher Einkommen überproportional entlasten. Diese Annahme scheint in Anbetracht der erheblichen Senkung des Spitzensteuersatzes von $45 \%$ auf 35\% berechtigt zu sein.

Hinsichtlich der Veränderung des verfügbaren Nettoeinkommens ergibt sich für Bezieher höherer Einkommen sowohl eine absolut als auch eine relativ höhere Entlastung als für die übrigen Steuerzahler. ${ }^{21}$ Bei geringen Einkommen hat die niedrige Steuerlast für die Höhe des Nettoeinkommens allerdings schon beim gegenwärtigen Formeltarif nur eine untergeordnete Bedeutung. Daher kann eine Steuertarifsenkung das Nettoeinkommen von Geringverdienern auch nicht in relevantem Maße verändern. Aus diesem Grunde erscheint es zweckmäßiger bei der Verteilungsanalyse alternativer Steuerkonzepte auf die Steuerlastverteilung abzustellen. ${ }^{22}$ Ein Vergleich der Steuerlastverteilung ergibt ein anderes Ergebnis als die Analyse von Nettoeinkommen. Die bei Anwendung des FDP-3-Stufentarifs im Vergleich zum Formeltarif 2010 insgesamt erheblich niedrigere Steuerlast wird in stärkerem Maße von den Steuerpflichtigen mit den höchsten Einkommen getragen, so dass die Steuerlastverteilung deutlich ungleicher ausfällt.

68,7 Mrd. $€$ (vgl. Neumann, Peichl, Schneider und Siegloch 2009). Das RWI ermittelt auf Basis der Daten des Scientific Use Files der Einkommensteuerstatistik 2001 (FAST 2001) für den FDP-3Stufentarif im Vergleich zum Formeltarif 2009 Steuermindereinnahmen i.H.v. rund 67,8 Mrd. $€$ und eine durchschnittliche Steuerentlastung i.H.v. 32,97\% (vgl. RWI 2009, S. 25 und 30).

21. Vgl. auch Neumann, Peichl, Schneider und Siegloch (2009), S. 810.

22. Vgl. zur Wahl dieses Maßstabs ausführlich Maiterth und Müller (2009) und Abschnitt 3. 


\section{Aufkommens- und Verteilungsfolgen des Ersatzes des deutschen einkommensteuerlichen Formeltarifs durch einen Stufentarif}

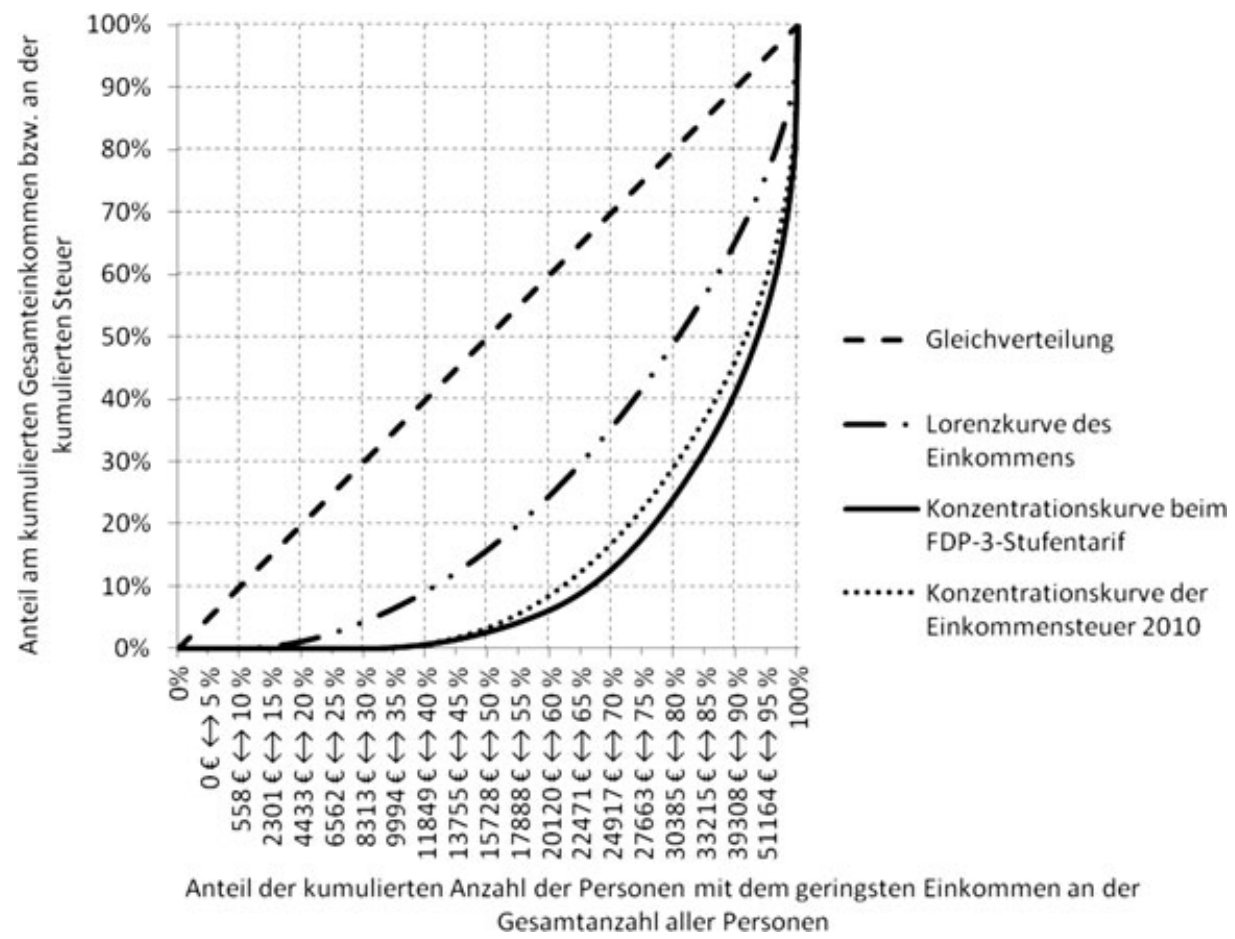

Abbildung 4 Lorenzkurve des Einkommens und Konzentrationskurve der Einkommensteuerlast 2010 sowie des FDP-3-Stufen-Tarifs.

Abbildung 4 enthält die Lorenzkurve des Einkommens sowie die Konzentrationskurven der Einkommensteuer nach dem Formeltarif 2010 und nach dem FDP-3-Stufentarif. Es wird deutlich, dass durch den FDP-3-Stufentarif die Einkommensteuer erheblich ungleicher zulasten einkommensstarker Personen verteilt ist als beim Formeltarif 2010.

Abbildung 4 zeigt, dass die aggregierte relative Einkommensteuerlast beim FDP3-Stufentarif in Richtung der Bezieher hoher und höchster Einkommen verschoben wird. Tragen die 60\% der Personen mit den geringsten Einkommen beim Formeltarif 2010 noch gut 8,6\% der aggregierten Steuerlast, so trägt diese Gruppe beim FDP-3Stufentarif nur noch rund 6,2\% der Steuerlast. Dagegen steigt die relative Steuerlast der 10\% einkommensstärksten Personen von etwa 53,5\% auf 58,6\%. Diese steigende Ungleichheit kommt auch deutlich im Kakwani-Progressionsmaß zum Ausdruck, welches von 0,212 beim aktuell geltenden Formeltarif $2010 \mathrm{um}$ mehr als 18\% auf 0,251 beim FDP-3-Stufentarif steigt.

Eine genauere Identifikation der Gewinner und Verlierer des FDP-3-Stufentarifs wird anhand der Abbildung 5 möglich, in der die Änderung des individuellen Durchschnittssteuersatzes mit der Änderung des empirisch ermittelten aggregierten Durchschnittssteuersatzes verglichen wird.

Unter der Anwendung des FDP-3-Stufentarifs ergibt sich im Vergleich zum Formeltarif 2010 empirisch eine aggregierte Durchschnittsentlastung i.H.v. 31,91\%. Der Durchschnittssteuersatz sinkt - wie bereits ausgeführt - von 19,80\% um 6,32 Prozentpunkte auf 13,48\%. Es zeigt sich, dass diejenigen Personen, deren 
Henriette Houben, Ralf Maiterth und Heiko Müller

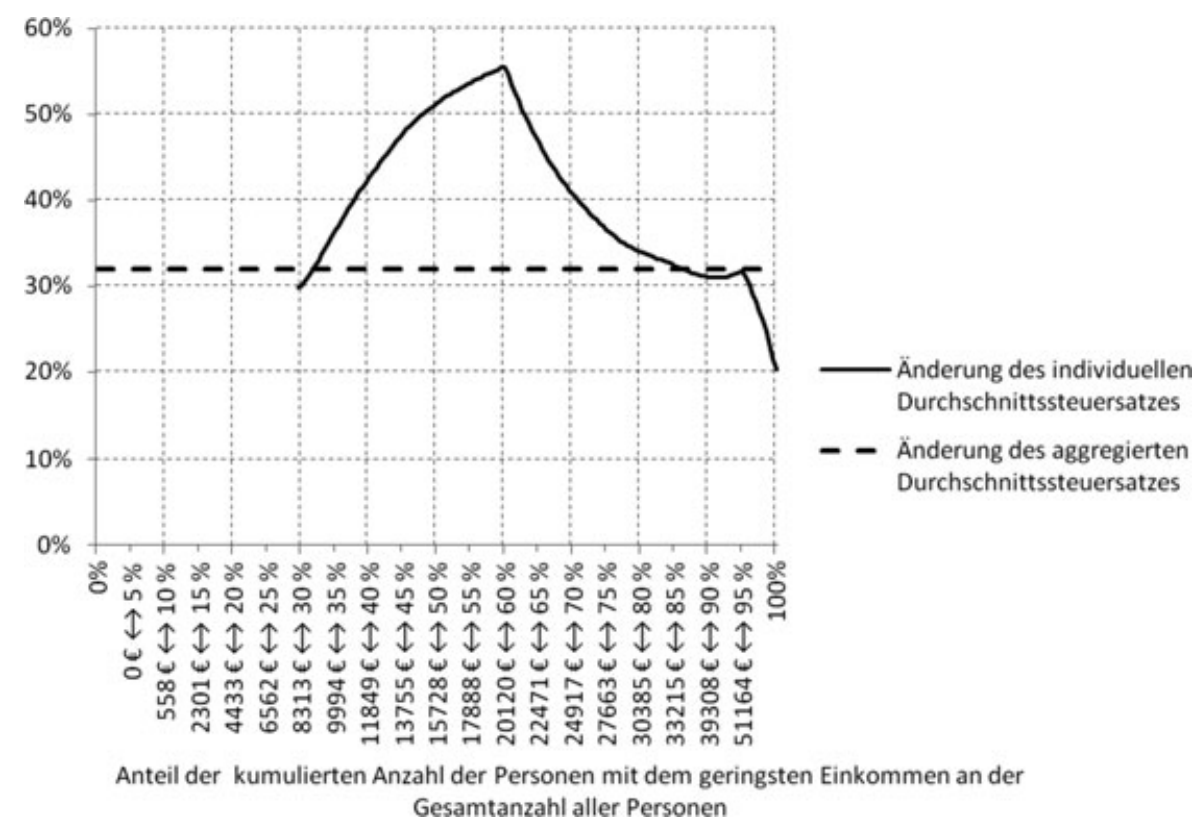

Abbildung 5 Gewinner und Verlierer des FDP-3-Stufentarifs.

Einkommen zwischen den Perzentilgrenzen i.H.v. $8.741 € €$ und $34.196 €$ liegen, gegenüber der durchschnittlichen Steuerentlastung aller Steuerpflichtigen eine überdurchschnittliche Steuerentlastung erfahren, während Bezieher über $34.196 €$ liegender Einkommen lediglich unterdurchschnittlich entlastet werden.

In einem Tarifvergleich, der ausschließlich auf das zu versteuernde Einkommen und den Tarif gemäß $\S 32 \mathrm{a}$ EStG abstellt, zeigt sich, dass die höchste relative Entlastung i.H.v. 55,6\% ein zu versteuerndes Einkommen i.H.v. $20.000 €$ (1. Stufenobergrenze des FDP-3-Stufentarifs) und die geringste ein $\mathrm{zu}$ versteuerndes Einkommen i.H.v. 250.730 € (Ende der ersten Proportionalzone des Formeltarifs) mit 18,7\% erfährt. Die Spreizung zwischen der höchsten und niedrigsten relativen Entlastung der unter dem FDP-3-Stufentarif Steuerbelasteten beträgt im Tarifvergleich folglich beachtliche 36,9 Prozentpunkte. Unabhängig von den immensen Aufkommenswirkungen kann dem FDP-3-Stufentarif jedoch attestiert werden, dass ein wesentlicher Bereich niedriger und mittlerer Einkommen überdurchschnittlich entlastet werden.

\subsection{Aufkommens- und Verteilungswirkungen des FDP-5-Stufentarifs}

Der auf dem 61. FDP-Parteitag vorgestellte 5-Stufentarif weist nach dem Grundfreibetrag i.H.v $8.004 €$ einen Eingangssteuersatz i.H.v. $14 \%$ auf. Im Einkommensbereich zwischen $12.501 €$ und $35.000 €$ kommt ein Steuersatz i.H.v. 25\% zur Anwendung. In der darauf folgenden Tarifstufe gilt bis zu einem Einkommen i.H.v. $53.000 €$ ein Steuersatz i.H.v. 35\%. Der $53.000 €$ übersteigende Betrag unterliegt bis $250.730 €$ einem Steuersatz i.H.v. 42\%. Ab einem Einkommen i.H.v. 250.731 € beginnt der Bereich des so genannten „Reichensteuersatzes“ i.H.v. 


\section{Aufkommens- und Verteilungsfolgen des Ersatzes des deutschen einkommensteuerlichen Formeltarifs durch einen Stufentarif}

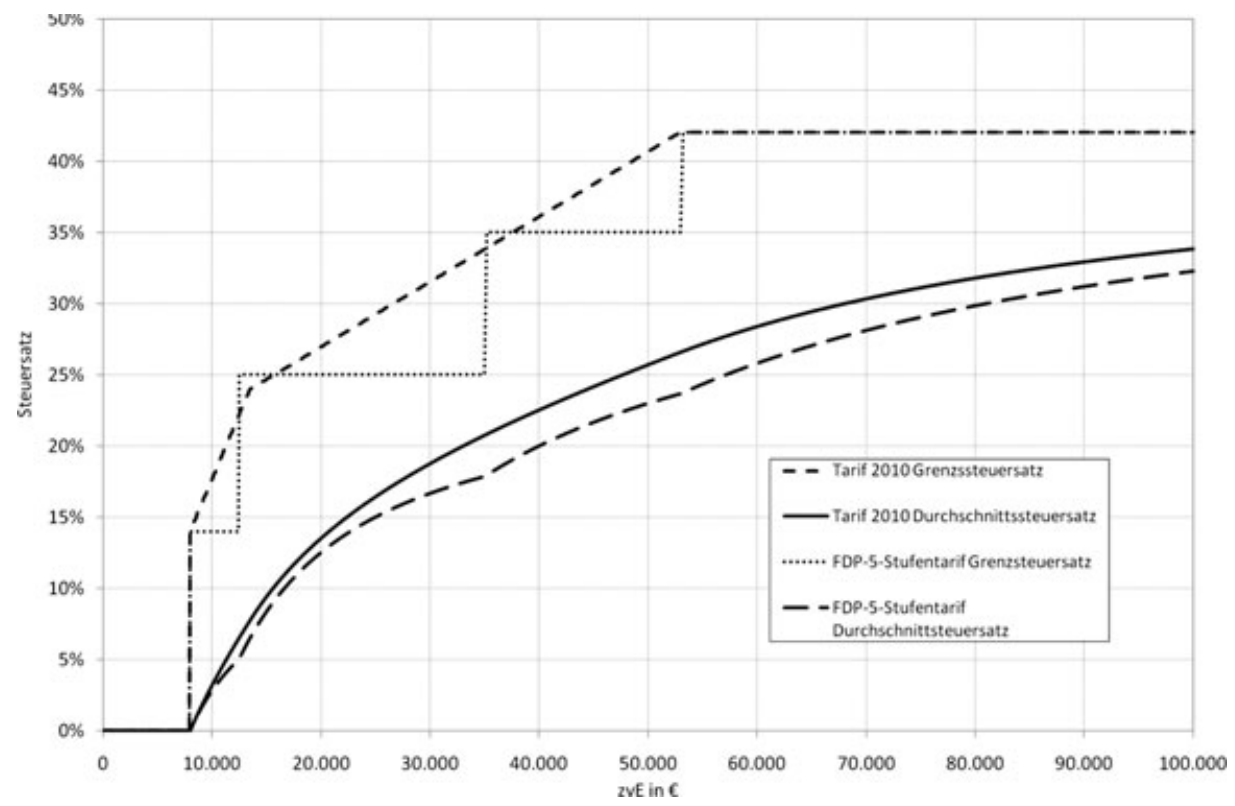

Abbildung 6 Grenz- und Durchschnittssteuersatz beim Formeltarif 2010 und FDP-5-Stufentarif.

45\%. Abbildung 6 zeigt sowohl die Grenz- als auch die Durchschnittssteuersätze des Einkommensteuertarifs 2010 und des FDP-5-Stufentarifs.

Der FDP-5-Stufentarif führt im Vergleich zum Formeltarif 2010 zu Steuermindereinnahmen von rund 16,3 Mrd. €. Dies sind 8,17\% des Aufkommens des Formeltarifs 2010. Der empirische Durchschnittssteuersatz sinkt um 1,62 Prozentpunkte bzw. 8,17\% auf 18,18\%. Auch der FDP-5-Stufentarif führt im Vergleich zum Formeltaif 2010 zu einer ungleicheren Verteilung der Steuerlast. Das Kakwani-Progressionsmaß steigt von 0,212 um rund 2,8\% auf 0,218. Ein differenzierteres Bild der Verteilungswirkung ergibt die einkommensbezogene Gewinner-/Verlierer-Analyse in Abbildung 7.

Im Vergleich zum Formeltarif 2010 ergeben sich unter Anwendung des FDP5-Stufentarifs empirisch drei Verlierer- und zwei Gewinnergruppen. Personen, deren Einkommen unter $9.448 €$, zwischen $17.217 €$ und $24.174 € €$ oder über $66.726 €$ liegt, werden durch die Tarifänderung unterdurchschnittlich entlastet. Hingegen werden die Personen, deren Einkommen zwischen 9.448 € und $17.217 €$ liegt, überdurchschnittlich stark entlastet. Der ausschließlich auf die Tarifvorschrift des $\S 32$ a EStG bezogene Tarifvergleich zeigt, dass wiederum an der 1. Stufenobergrenze bei einem zu versteuernden Einkommen i.H.v. $12.500 €$ die höchste Steuerentlastung i.H.v. 22,6\% erreicht wird. Ebenfalls zu den Gewinnern gehören die Personen, deren Einkommen zwischen den Perzentilgrenzen i.H.v. $24.174 € €$ und $66.726 €$ liegt. In diesem Bereich beträgt die im Tarifvergleich ermittelte maximale Steuerentlastungen an der 3. Stufenobergrenze bei einem zu versteuernden Einkommen i.H.v. $35.000 €$ etwa 13,8\%. An der 4. Stufenobergrenze i.H.v. $53.000 €$ beläuft sich die überdurchschnittliche Steuerentlastung nur noch auf $10,9 \%$. Mit weiter steigenden Einkommen nimmt die relative Steuerentlastung stetig ab und strebt gegen null. Somit beträgt beim Vergleich des FDP-5-Stufentarifs 


\section{Henriette Houben, Ralf Maiterth und Heiko Müller}

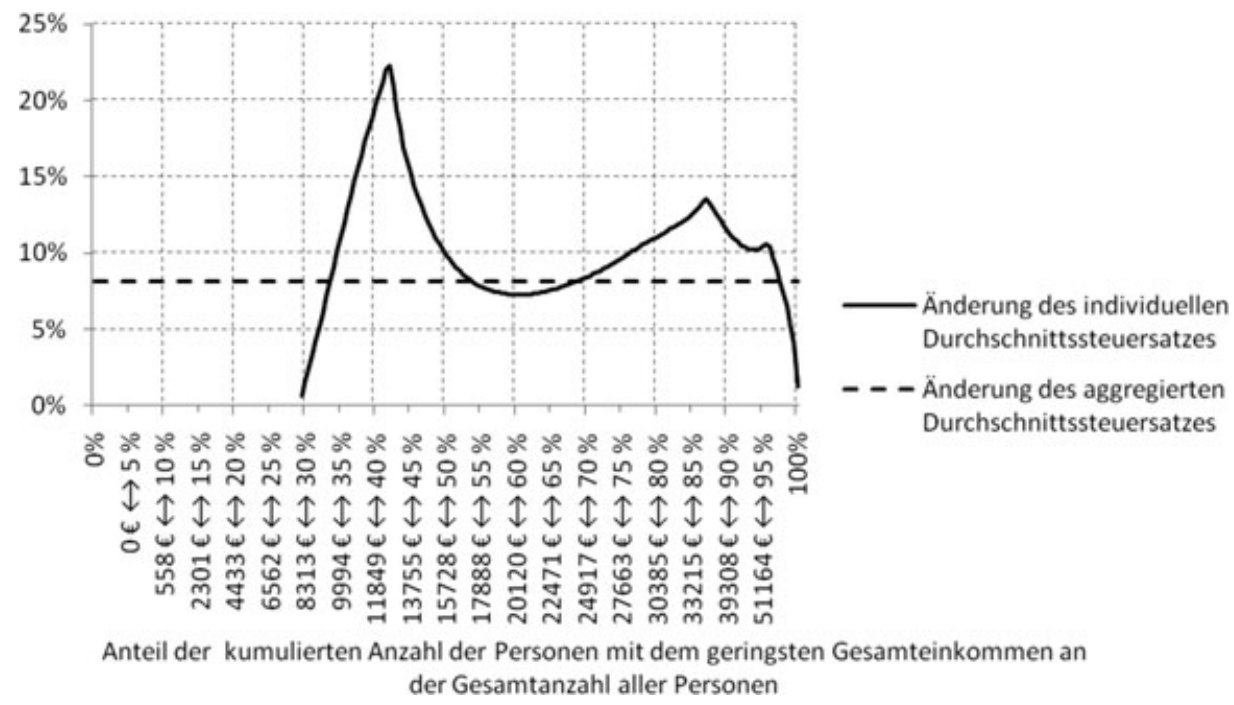

Abbildung 7 Gewinner und Verlierer des FDP-5-Stufentarifs.

mit dem Formeltarif 2010 die Spreizung zwischen der maximalen und minimalen relativen Steuerentlastung über 22 Prozentpunkte.

Die relativen Entlastungswirkungen des FDP-5-Stufentarifs unterscheiden sich erheblich von denen des FDP-3-Stufentarifs. Der FDP-3-Stufentarif entlastet Bezieher niedriger bis mittlerer Einkommen durchgängig überproportional und Bezieher hoher Einkommen unterproportional. Dagegen werden beim FDP-5Stufentraif genau diejenigen Einkommen, die beim FDP-3-Stufentarif am stärksten entlastet wurden, lediglich unterdurchschnittlich entlastet. Eine Person mit einem zu versteuernden Einkommen i.H.v. $20.000 €$ erfährt beim FDP-3-Stufentarif mit 55,6\% eine um 23,7 Prozentpunkte höhere Entlastung als der empirische Durchschnitt. Dagegen liegt die relative Entlastung für dieses Einkommen beim FDP-5-Stufentarif mit 7,3\% unter dem empirischen Durchschnitt i.H.v. 8,2\%. Im Gegensatz zum FDP-3-Stufentarif erhält beim FDP-5-Stufentarif auch ein erheblicher Teil der 10\% Einkommensstärksten eine überdurchschnittliche Entlastung. Somit bewirkt der FDP-5-Stufentarif nicht nur erheblich geringere Steuermindereinnahmen, sondern auch wesentlich andere Verteilungswirkungen als der FDP-3-Stufentarif. Dem FDP-5-Stufentarif kann nicht uneingeschränkt attestiert werden, dass er mittlere Einkommen überdurchschnittlich entlastet, da Einkommen zwischen $17.217 €$ und $24.174 €$ unterdurchschnittlich entlastet werden. Da auch der FDP-5-Stufentarif mit rund 16,3 Mrd. € beachtliche Steuermindereinnahmen verursacht, werden nachfolgend alternative Stufentarife dargestellt, deren Aufkommenswirkungen lediglich moderat ausfallen.

\section{Alternative Stufen-Tarife}

\subsection{Prämissen bei der Tarifgestaltung}

Da ein nur dreistufiger Tarif im Vergleich zum bisher gültigen linearprogressiven Formeltarif hinsichtlich der relativen Steuerlaständerung zwischen verschiedenen Einkommensgruppen zu stark unterschiedlichen Entlastungen führt, 


\section{Aufkommens- und Verteilungsfolgen des Ersatzes des deutschen einkommensteuerlichen Formeltarifs durch einen Stufentarif}

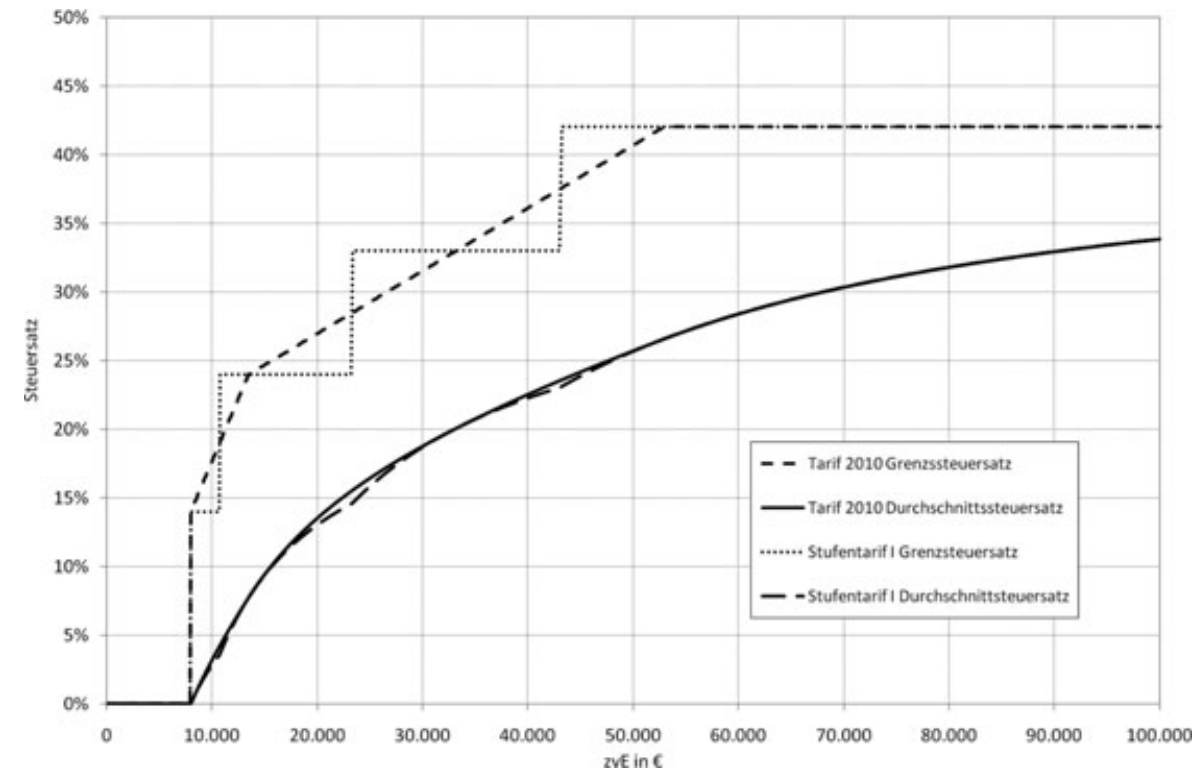

Abbildung 8 Grenz- und Durchschnittssteuersatz Formeltarif 2010 versus Stufentarif I.

werden nachfolgend - analog zum aktuellen FDP-Vorschlag - fünfstufige Tarife entwickelt, bei denen sich durch eine entsprechende Tarifgestaltung derartige Unterschiede erheblich verringern. Zudem wird bei der Tarifgestaltung von der Prämisse ausgegangen, dass die Personen in den untersten Einkommensklassen keine nennenswerte Mehrbelastung gegenüber dem Formeltarif 2010 erfahren sollen. Demzufolge wird - wie bereits bei den beiden FDP-Tarifvorschlägen - ein Grundfreibetrag zur Freistellung des Existenzminimums i.H.v. $8.004 € €$ berücksichtigt. Primäres Ziel aller vorgestellten Alternativtarife ist jedoch die Aufkommensstabilisierung.

\subsection{An den Formeltarif 2010 angelehnter Stufentarif (Stufentarif I)}

Der Stufentarif I orientiert sich stark an der Tarifgestalt des linear-progressiven Formeltarifs 2010. Der Eingangssteuersatz des Stufentarifs I beträgt 14\%. Die Obergrenze der ersten Tarifstufe entspricht mit $10.737 €$ der Intervallmitte der ersten Progressionszone des Tarifs 2010. In Anlehnung an den Eingangssteuersatz der zweiten Progressionszone des Tarifs 2010 i.H.v. 23,97\% beträgt der Steuersatz der zweiten Tarifstufe 24\%. Die dritte Tarifstufe beginnt bei einem Einkommen i.H.v. $23.324 €$ und sieht einen Steuersatz i.H.v. 33\% vor. Der Spitzensteuersatz der ersten Proportionalzone des Formeltarifs 2010 i.H.v. 42\% wird übernommen und kommt bereits bei einem Einkommen i.H.v. $43.028 €$ zur Anwendung, wogegen dies beim Formeltarif 2010 erst ab einem Einkommen i.H.v. $52.882 €$ erfolgt. Die letzte Tarifstufe wurde derart gestaltet, dass analog zum Formeltarif 2010 der Spitzensteuersatz i.H.v. 45\% ab einem Einkommen i.H.v. $250.731 € €$ Anwendung findet.

Abbildung 8 lässt erkennen, dass die Durchschnittssteuerbelastung des Stufentarifs I im gesamten Einkommensbereich kaum von der Durchschnittssteuerbelastung des Tarifs 2010 abweicht. 


\section{Henriette Houben, Ralf Maiterth und Heiko Müller}

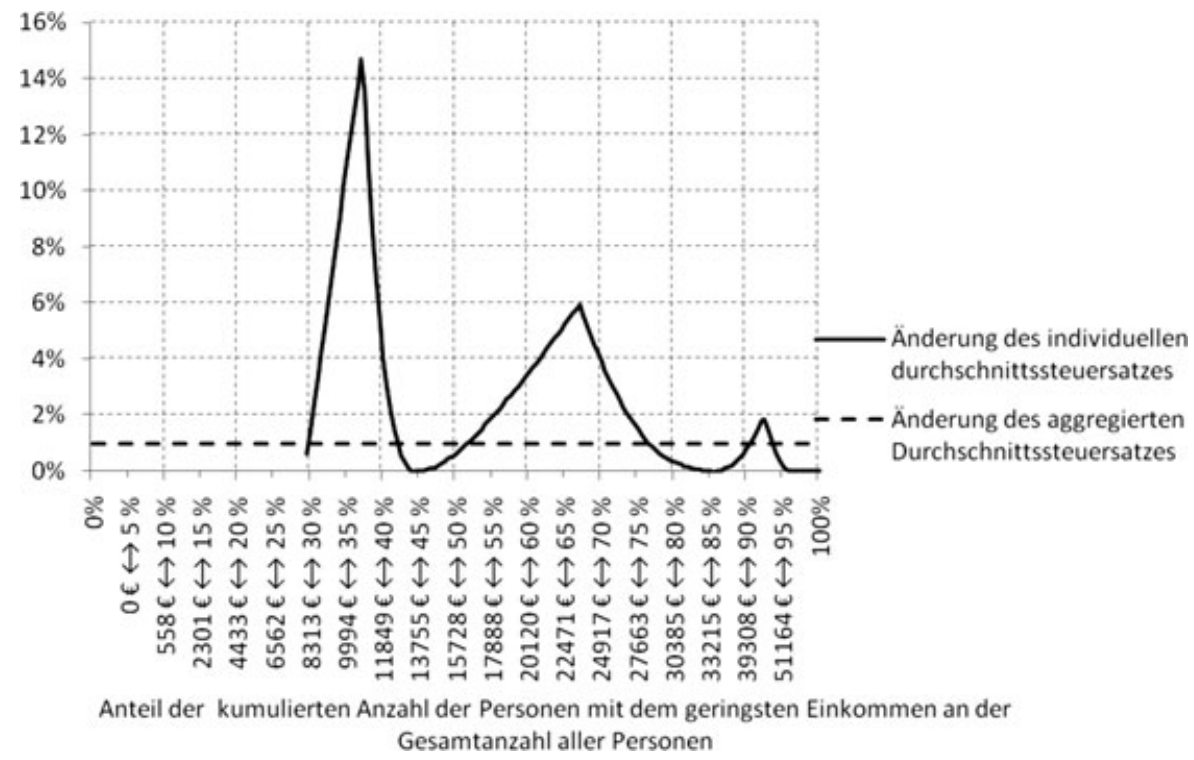

Abbildung 9 Gewinner und Verlierer des Stufentarifs I.

Trotz der starken Orientierung am Formeltarif 2010 führt der Stufentarif I im Vergleich mit dem Formeltarif 2010 zu Steuermindereinnahmen i.H.v. 1,89 Mrd. $€$ und damit korrespondierend zu einer geringfügigen Verringerung des empirischen aggregierten Durchschnittssteuersatzes von 19,80\% auf 19,61\%. Der leichte Anstieg des Kakwani-Progressionsmaßes von 0,212 beim Formeltatarif 2010 um 1,6\% auf 0,215 beim Stufentarif I zeigt eine leichte Zunahme der Ungleichverteilung der Einkommensteuerlast an. Verteilungswirkungen dieses Ausmaßes haben sich bereits bei der Tarifänderung 2009/2010 ergeben und scheinen im politischen Prozess akzeptiert zu werden.

Abbildung 9 zeigt wiederum, welche Gruppen bei der Einführung des Stufentarifs I über- bzw. unterdurchschnittlich entlastet werden.

Der einkommensbezogene Vergleich der Veränderung der Durchschnittssteuerbelastung zeigt, dass insbesondere diejenigen Steuerpflichtigen, deren Einkommen sich unmittelbar in dem Bereich der ersten drei Stufengrenzen befindet, überproportional entlastet werden. Für die übrigen Steuerpflichtigen ändert sich die Steuerbelastung dagegen nur unwesentlich. In einigen Tarifbereichen kommt es zu geringfügig höheren Durchschnittssteuerbelastungen, wobei der jeweilige Durchschnittssteuersatz des Stufentarifs I nie um mehr als 0,02 Prozentpunkte über dem Durchschnittssteuersatz des Formeltarifs 2010 liegt.

Der Tarifvergleich zeigt, dass die maximale Spreizung zwischen der höchsten relativen Entlastung i.H.v. 15,11\% bei einem $\mathrm{zu}$ versteuernden Einkommen i.H.v. $10.737 €$ und einer relativen Mehrbelastung i.H.v. 0,06\% bei einem zu versteuernden Einkommen i.H.v. $32.432 €$ bei etwas mehr als 15 Prozentpunkten liegt und deutlich geringer als bei den FDP-Tarifen ausfällt. Allerdings kann auch dem Stufentarif I nicht attestiert werden, dass er ausschließlich mittlere Einkommen überproportional entlastet. Zudem dürfte der hier vorgestellte Stufentarif I nicht dem FDP-Ideal eines einfachen und transparenten Tarifs entsprechen. 


\section{Aufkommens- und Verteilungsfolgen des Ersatzes des deutschen einkommensteuerlichen Formeltarifs durch einen Stufentarif}

Daher wird nachfolgend ein Stufentarif mit runden und damit einprägsameren Einkommensgrenzen und Steuersätzen vorgestellt.

\subsection{Ein „einfacher" Stufentarif (Stufentarif II)}

Ein einfacher Stufentarif mit einer einprägsamen Tarifstruktur, einem Grundfreibetrag i.H.v. $8.004 €$ und einem niedrigen Eingangssteuersatz erfordert eine immense Absenkung des Beginns des Anwendungsbereichs des Spitzensteuersatzes. Werden beispielsweise ein Eingangssteuersatz i.H.v. 10\% bis $\mathrm{zu}$ einem $\mathrm{zu}$ versteuernden Einkommen i.H.v. $10.000 €$ und Steuersätze i.H.v. $20 \%$ bis $20.000 €$, $30 \%$ bis $30.000 €$ und $40 \%$ bis $40.000 €$ gewählt, dann muss der Spitzensteuersatz i.H.v. $45 \%$ bereits ab einem $\mathrm{zu}$ versteuernden Einkommen i.H.v. $40.000 €$ greifen, um zumindest näherungsweise Aufkommensneutralität zu gewährleisten. ${ }^{23}$ Ein derartiger Tarif dürfte insbesondere wegen des frühen Einsetzens des Spitzensteuersatzes politisch nicht durchsetzbar sein. Zudem verschiebt sich die Einkommensteuerlast erheblich zu Lasten von Beziehern mit Einkommen ab etwa $35.000 €$, die bereits beim Tarif 2010 den Großteil des Einkommensteueraufkommens bestreiten. ${ }^{24}$

Nachfolgend wird ein einfacher Stufentarif (Stufentarif II) diskutiert, der mit einem Spitzensteuersatz i.H.v. 45\% ab einem Einkommen von $120.000 €$ politisch akzeptabel erscheint. Der Grundfreibetrag beträgt unverändert $8.004 €$. Bis zu einem Einkommen i.H.v. $10.000 €$ kommt ein Steuersatz i.H.v. 15\% zur Anwendung. Die darauf folgende Stufe mit einem Steuersatz i.H.v. 20\% endet bei $20.000 €$. Der Steuersatz der dritten Stufe i.H.v. 35\% gilt bis zu einem Einkommen i.H.v. 50.000 $€$. Darüber hinausgehende Einkommensteile werden unterhalb von $120.000 €$ mit einem Steuersatz i.H.v. 40\% und danach mit 45\% besteuert.

Der Stufentarif II würde im Vergleich zum Formeltarif 2010 zu Steuermindereinnahmen i.H.v. rund 1,36 Mrd. € führen. Unter Anwendung des Stufentarifs II sinkt der aggregierte Durchschnittssteuersatz um 0,14 Prozentpunkte bzw. 0,68\% auf 19,66\%. Im Vergleich zum Formeltarif 2010 nimmt die Ungleichverteilung der Einkommensteuerlast geringfügig zu. Das Kakwani-Progressionsmaß steigt um 3,2\% auf 0,219.

Der Vergleich der Durchschnittssteuersätze in Abbildung 10 zeigt, dass bei der Einführung des Stufentarifs II Personen mit einem Einkommen zwischen etwa $9.100 €$ und $26.100 € \mathrm{im}$ Vergleich zum Stufentarif 2010 geringer belastet werden. Dagegen erfahren Personen, deren Einkommen zwischen 26.100 € und $49.100 €$ liegt, eine höhere Steuerbelastung. Dies gilt auch für Einkommen über $167.700 €$. Ein genaueres Bild zeichnet die empirische Gewinner-Verlierer-Analyse in Abbildung 11.

Beim Stufentarif II gibt es keine einheitliche Gewinner-/Verliererstruktur. Vielmehr existieren mehrere Gruppen, die über- bzw. unterdurchschnittlich entlastet oder sogar belastet werden. Augenfällig ist, dass beim Stufentarif II auch niedrige Einkommen unterdurchschnittlich entlastet und sogar belastet werden. Aus Abbildung 11 wird deutlich, dass die Anhebung des Eingangssteuersatzes um einen Prozentpunkt auf 15\% Geringverdiener relativ stärker belastet als

23. Dieser Tarif bewirkt im Vergleich zum Formeltarif 2010 Steuermindereinnahmen i.H.v. 2,3 Mrd. $€$. 24. Vgl. ausführlich dazu Houben, Maiterth und Müller (2010), S. 20 ff. 


\section{Henriette Houben, Ralf Maiterth und Heiko Müller}

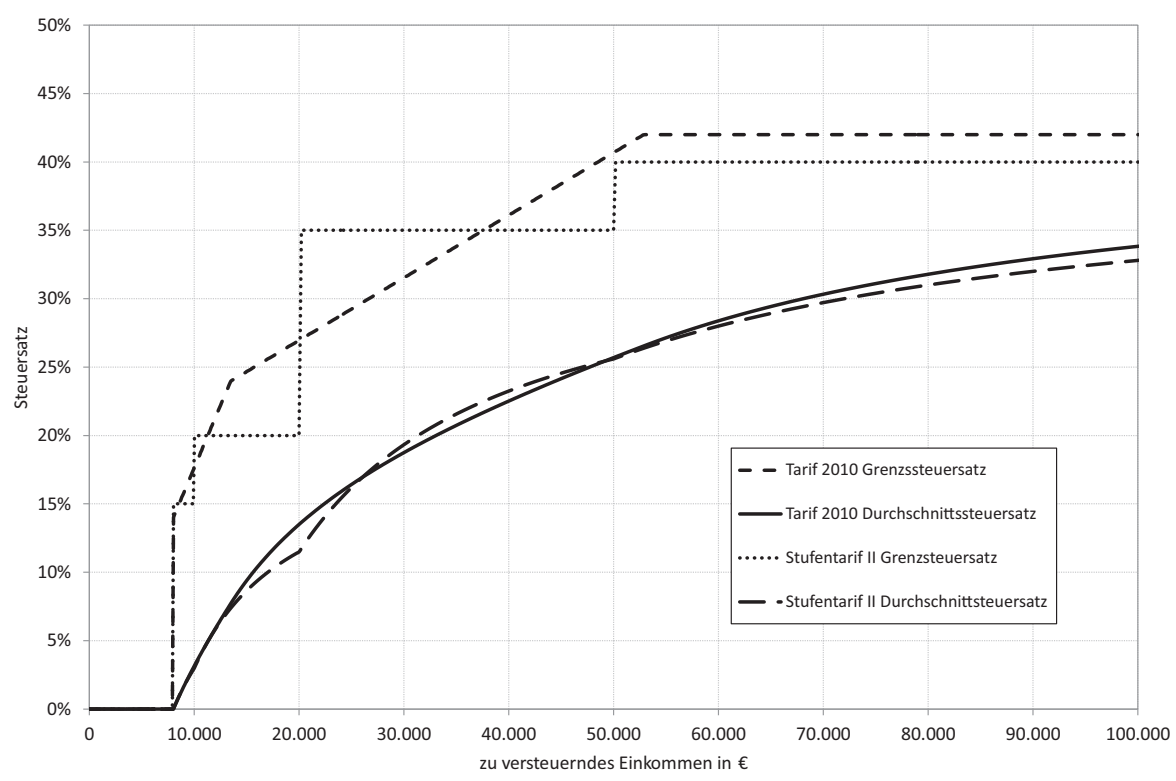

Abbildung 10 Grenz- und Durchschnittssteuersatz des Tarifs 2010 versus Stufentarif II.

der Tarif 2010. Allerdings steigt die absolute Einkommensteuerlast in diesem „Verlierersegment" nur um jeweils maximal $3 €$. Zudem wird deutlich, dass Personen mit einem Einkommen um $11.000 €$ zwar nach der Tarifänderung geringfügig weniger Steuern zahlen als beim Formeltarif 2010, jedoch wird diese Gruppe unterdurchschnittlich entlastet. Ein Tarifvergleich zeigt, dass wiederum ein zu versteuerndes Einkommen i.H.v. $20.000 €$ die höchste relative Entlastung i.H.v. $14,9 \%$ erfährt. Beim Stufentarif II werden auch Steuerpflichtige mit einem zu versteuernden Einkommen zwischen ca. $53.450 €$ und $154.700 €$ überdurchschnittlich entlastet. Somit erfahren auch Personen aus dem Bereich des 96ten bis 99,5ten Perzentil eine überdurchschnittliche Entlastung.

Da beim Stufentarif II Personen mit einem Einkommen zwischen den Perzentilgrenzen i.H.v. $11.849 €$ und $25.715 €$ überdurchschnittlich entlastet werden und Einkommen zwischen $25.716 €$ und $55.648 €$ eine unterdurchschnittliche Entlastung erfahren oder sogar zusätzlich belastet werden, liegt es nahe, unter Wahrung der Aufkommensneutralität und der Transparenz zur Entlastung der mittleren und höheren Einkommen den Steuersatz der Tarifstufe zwischen $10.000 €$ und $20.000 €$ von $20 \%$ auf $25 \%$ zu erhöhen und den Steuersatz der Tarifstufe zwischen $20.000 €$ und $50.000 €$ von $35 \%$ auf $30 \%$ zu senken. Der sich daraus ergebende alternative Stufentarif würde mit 1,05 Mrd. € nur moderate Aufkommenseinbußen verursachen, allerdings würde sich das Gewinner/Verlierer-Bild drastisch ändern. Der angestrebte Effekt, niedrige und mittlere Einkommen zu entlasten, würde nicht erreicht. ${ }^{25}$ Vielmehr würden im Vergleich zum Formeltarif 2010 diejenigen Personen sogar mehr belastet, deren Einkommen zwischen den Perzentilgrenzen i.H.v. $10.173 €$ und $36.457 €$ liegt. Dies sind ca. 52,5\% aller Personen. Dagegen würden Bezieher von Einkommen zwischen

25. Vgl. ausführlich dazu Houben, Maiterth und Müller (2010), S. 25 f. 


\section{Aufkommens- und Verteilungsfolgen des Ersatzes des deutschen einkommensteuerlichen Formeltarifs durch einen Stufentarif}

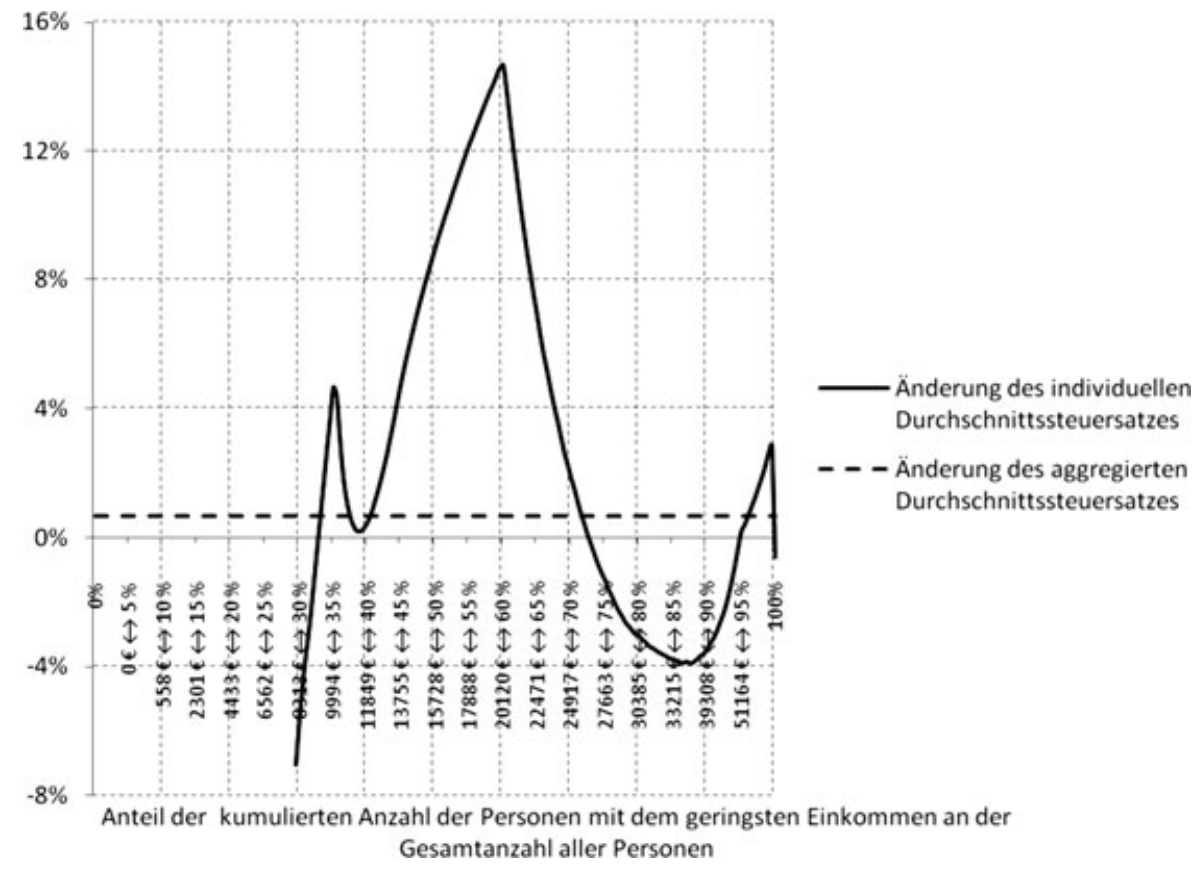

Abbildung 11 Gewinner und Verlierer des Stufentarifs II.

$37.000 €$ und $188.400 €$ überdurchschnittlich entlastet. Eine derartige Gewinner-/ Verlierer-Struktur dürfte politisch nicht durchsetzbar sein. Dies zeigt jedoch, dass die Zielsetzungen Aufkommensneutralität, Entlastung insbesondere niedriger und mittlerer Einkommen sowie runde und damit einprägsamere Einkommensgrenzen und Steuersätze nur schwer umsetzbar sind. Mit den als transparent empfundenen Einkommensgrenzen und Steuersätzen wird die Erreichbarkeit der Aufkommensund Verteilungsziele entscheidend begrenzt.

\section{Fazit}

Ein Ersatz des deutschen Einkommensteuer-Formeltarifs durch einen Stufentarif wird insbesondere mit dem Argument der Steuervereinfachung gefordert. Ein unbestreitbarer Vorteil eines Stufentarifs besteht darin, dass die entscheidungsrelevanten Grenz- und Differenzsteuersätze in der Regel unmittelbar dem Steuertarif entnommen werden können. Im direkt progressiven Bereich des Formeltarifs müssen Grenz- und Differenzsteuersätze dagegen rechnerisch ermittelt werden, was dem Großteil der Steuerpflichtigen Probleme bereiten dürfte.

Die vorliegende Untersuchung zeigt, dass mit dem FDP-3-Stufentarif durchaus auch das Ziel erreicht würde, mit einem einfachen Stufentarif niedrige und mittlere Einkommen überdurchschnittlich $\mathrm{zu}$ entlasten. Allerdings ist der FDP3-Stufentarif mit einer enormen Steuersenkung verbunden, die im Vergleich zum Formeltarif $2010 \mathrm{zu}$ Steuermindereinnahmen i.H.v. 63,6 Mrd. $€$ führt. Im Hinblick auf die aktuelle Lage der öffentlichen Haushalte hat die FDP jüngst einen 5-Stufentarif vorgestellt, der lediglich Steuermindereinnahmen i.H.v. 16,3 Mrd. $€$ bewirkt. Die Verteilungswirkungen dieses 5-Stufentarifs unterscheiden 
sich gravierend von denen des FDP-3-Stufentarifs. Der FDP-5-Stufentarif bewirkt nicht durchgängig eine überdurchschnittliche Entlastung niedriger und mittlerer Einkommen. Zudem werden auch weite Teile der Bezieher höherer und hoher Einkommen überdurchschnittlich entlastet. Ein 5-Stufentarif, der bei lediglich moderaten Aufkommenswirkungen strukturell ähnliche Verteilungswirkungen wie der FDP-3-Stufentarif zeitigt, geht mit einer massiven Absenkung der Tarifgrenze des Spitzensteuersatzes i.H.v. $45 \%$ auf $40.000 €$ und einer stärkeren Belastung von Einkommen ab etwa $35.000 €$ einher. Obgleich dies lediglich die 13\% Einkommensstärksten betrifft, erscheint es fraglich, ob Einkommen in der Größenordnung von $35.000 €$ bereits als hoch wahrgenommen werden und die massive Absenkung des Anwendungsbereichs des Spitzensteuersatzes politisch durchsetzbar ist.

Ein Stufentarif, der gegenüber dem Tarif 2010 nur geringe Aufkommensund Verteilungswirkungen bewirkt, lässt sich nur zu Lasten der Einfachheit der Tarifstruktur erreichen. Möglicherweise sind Verteilungseffekte auch dafür verantwortlich, dass bei den international üblichen Stufentarifen einprägsame Tarifstufen-Steuersatz-Strukturen eher selten sind. Als Beispiel seien die USA oder das Vereinigte Königreich genannt.

Soll ein Stufentarif für die allgemeine Wahrnehmung einfach im Sinne von leicht einprägsam und nachvollziehbar sein, dürfte dies nur mittels Tarifstufen und Steuersätzen erreichbar sein, die ein Vielfaches von $5.000 €$ bzw. $10.000 €$ und 5\% bzw. 10\% darstellen. Allerdings wird durch eine derartige Struktur die Flexibilität und Genauigkeit einer Tarifänderung hinsichtlich ihrer Aufkommens- und Verteilungswirkungen stark eingeschränkt. Ein Übergang vom linear-progressiven Formeltarif zu einem einfachen Stufentarif bewirkt schwer vermeidbare Verteilungseffekte in dem Sinne, dass bestimmte Einkommensklassen über- bzw. unterdurchschnittlich entlastet oder sogar belastet werden. Zudem muss der Spitzensteuersatz i.H.v. 45\% zur Aufkommenssicherung deutlich früher einsetzen als gegenwärtig.

Hinsichtlich des Ziels „Einfachheit der Besteuerung“ seien abschließend zwei Aspekte angemerkt. Das Ziel, einen einfachen und transparenten Stufentarif einzuführen, steht mit der im FDP-Bundesparteitagsbeschluss angestrebten - und durchaus gebotenen - kontinuierlichen Inflationsbereinigung des Steuertarifs in Konflikt. $^{26}$ Eine Inflationsanpassung führt zwangsläufig dazu, dass die runden Tarifstufengrenzen an die exakte Inflationsrate angepasst werden und damit die einfache Stufentarifstruktur verloren geht. Die alternativ mögliche Deflationierung der Bemessungsgrundlage widerspricht ebenfalls einer transparenten Besteuerung. Des Weiteren ist die von der FDP fast ausschließlich auf den Einkommensteuertarif fokussierte Steuervereinfachungsdiskussion sehr einseitig ausgerichtet. Mit der Änderung einer einzelnen Tarifvorschrift dürften kaum die erhofften Vereinfachungseffekte eintreten. Es ist unstrittig, dass die Kompliziertheit und Komplexität des Steuerrechts insbesondere aus der Ermittlung der Steuerbemessungsgrundlage resultieren und eine umfassende Steuervereinfachung bei der Gewinn- bzw. Einkommensermittlung ansetzen muss.

26. $\mathrm{Zu}$ den Inflationswirkungen des progressiven deutschen Einkommensteuertarifs vgl. Boss et al. (2008). 


\section{Aufkommens- und Verteilungsfolgen des Ersatzes des deutschen einkommensteuerlichen Formeltarifs durch einen Stufentarif}

\section{Literaturverzeichnis}

Bork, C. (2000), Steuern, Transfers und private Haushalte. Lang, Frankfurt am Main.

Boss, A., A. Boss und T. Boss (2008), Der deutsche Einkommensteuertarif: Wieder eine Wachstumsbremse?, Perspektiven der Wirtschaftspolitik 9, 102-124.

Boss, A., A. Boss und T. Boss (2009), Die Änderungen des Einkommensteuertarifs: Folgen für das Lohnsteueraufkommen und die Leistungsanreize, Kieler Working Paper Nr. 1529.

CDU/CSU/FDP (2009), Wachstum. Bildung. Zusammenhalt. Koalitionsvertrag zwischen CDU, CSU und FDP.

FDP (2008), Die gerechte Steuer: Einfach, niedrig und sozial. Das Nettokonzept der FDP. Beschluss des 59. Ordentlichen Bundesparteitags der FDP.

FDP (2010), Für faire Finanzbeziehungen zwischen Bürger und Staat. Ob als Steuerzahler oder als Transferempfänger - Leistung muss sich immer lohnen. Beschluss des 61. Ordentlichen Bundesparteitags der FDP.

Homburg, S., H. Houben und R. Maiterth (2008), Optimale Eigenfinanzierung der Personenunternehmen nach der Unternehmensteuerreform 2008/2009, Zeitschrift für betriebswirtschaftliche Forschung, 29-47.

Houben, H. und R. Maiterth (2008), Optimale Nutzung und Wirkungen von $\S 34 \mathrm{a}$ EStG, Steuer und Wirtschaft 85, 228-237.

Houben, H., R. Maiterth und H. Müller (2010), Aufkommens- und Verteilungsfolgen des Ersatzes des deutschen einkommensteuerlichen Formeltarifs durch einen Stufentarif, arqus-Working Paper Nr. 105.

Kakwani, N.C. (1977), Measurement of Progressivity: An International Comparison, Economic Journal 87, 71-80.

Kordsmeyer, V., (2004), Die Einkommensteuerstatistik als Mikrodatenfile, in: J. Merz und M. Zwick (Hrsg.), MIKAS, Mikroanalysen und Amtliche Statistik - Tagungsband zur gleichnamigen Veranstaltung im Februar 2002 in Lüneburg. Statistisches Bundesamt, Wiesbaden.

Lambert, S. et al. (1994), An Introduction to STINMOD: A Static Microsimulation Model, STINMOD, Technical Paper No. 1, Canberra.

Maiterth, R. und H. Müller (2009), Beurteilung der Verteilungswirkungen der „rot-grünen“ Steuerreform - Eine Frage des Maßstabs, Schmollers Jahrbuch 129, 375-390.

Merz, J. und M. Zwick (2002), Verteilungswirkungen der Steuerreform 2000/2005 im Vergleich zum „Karlsruher Entwurf“, Wirtschaft und Statistik 8, 729-740.

Müller, H. (2007), Ausmaß der einkommensteuerlichen Verlustverrechnung - Eine empirische Analyse der Aufkommens- und Verteilungswirkungen, Die Betriebswirtschaft 2, 179-200.

Neumann, D., A. Peichl, H. Schneider und S. Siegloch (2009), Die Steuerreformpläne der neuen Bundesregierung und Bürgergeld, Wirtschaftsdienst 89, 805-812.

RWI (2009), Auswirkungen der Steuerprogramme zur Bundestagswahl 2009 - Eine mikrodatenbasierte Analyse der Reformvorschläge von CDU/CSU, SPD und FDP, RWI Materialien Heft 55.

Sachverständigenrat (2009), Die Zukunft nicht aufs Spiel setzen. Jahresgutachten 2009/2010, Bonifatius Verlag, Paderborn.

Sutherland, H. (2000), Euromod - An Integrated European Benefit-Tax Model, in: A. Gupta und V. Kapur (Hrsg), Microsimulation in Government Policy and Forecasting. Elsevier, Amsterdam, 575-580.

van Essen, U., H. Kaiser und B.P. Spahn (1988), Verteilungswirkungen der Einkommensteuerreformen 1986 - 1990: Eine Simulationsstudie für die Bundesrepublik Deutschland auf Basis des Sozioökonomischen Panels, Finanzarchiv 46, 56-84.

Zwick, M. und K. Meyer (1998), Einzeldatenmaterial und Stichproben innerhalb der Steuerstatistiken, Wirtschaft und Statistik 50, 566-573. 


\begin{abstract}
A simplification of the German tax law is the major intend when proposing a graduated tax rate to replace the current German formula based tax scale. A plain tax rate structure requires catchy tax brackets (e.g. in $5.000 €$ or $10.000 €$ steps) and tax rates (e.g. tax rate differences of 5\% or 10\%). Our empirical analysis shows that such a graduated tax rate causes significant distributional effects and, depending on the particular form, affects different groups of tax payers in different ways. This holds in particular for the two graduated tax rate proposals from the German Liberal Party which additionally provoke substantial revenue losses. A graduated tax rate with little revenue and distributional effects compared to the current formula based tax scale lacks a plain structure and hence conflicts with the objective of tax simplification.
\end{abstract}




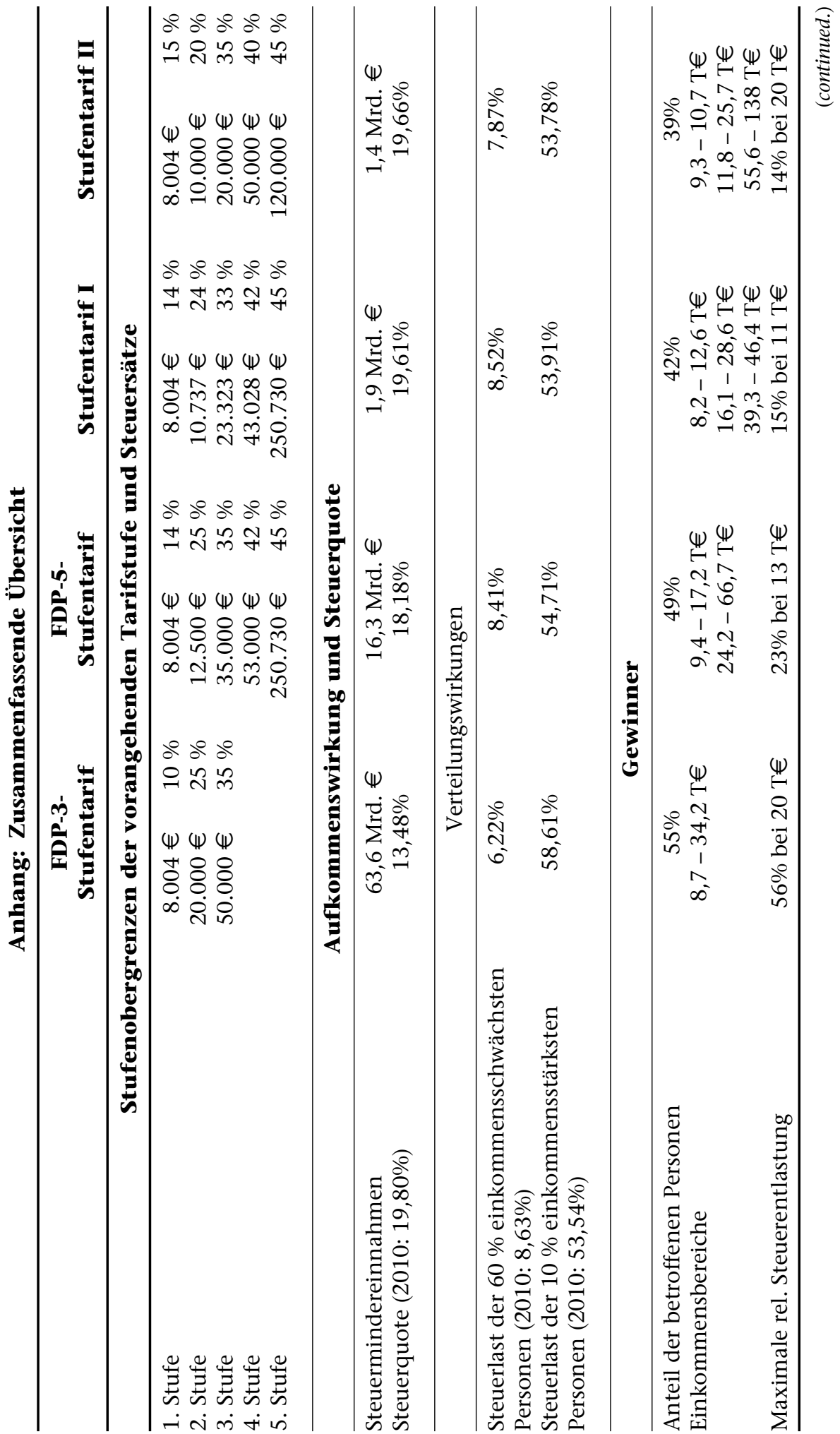


Henriette Houben, Ralf Maiterth und Heiko Müller

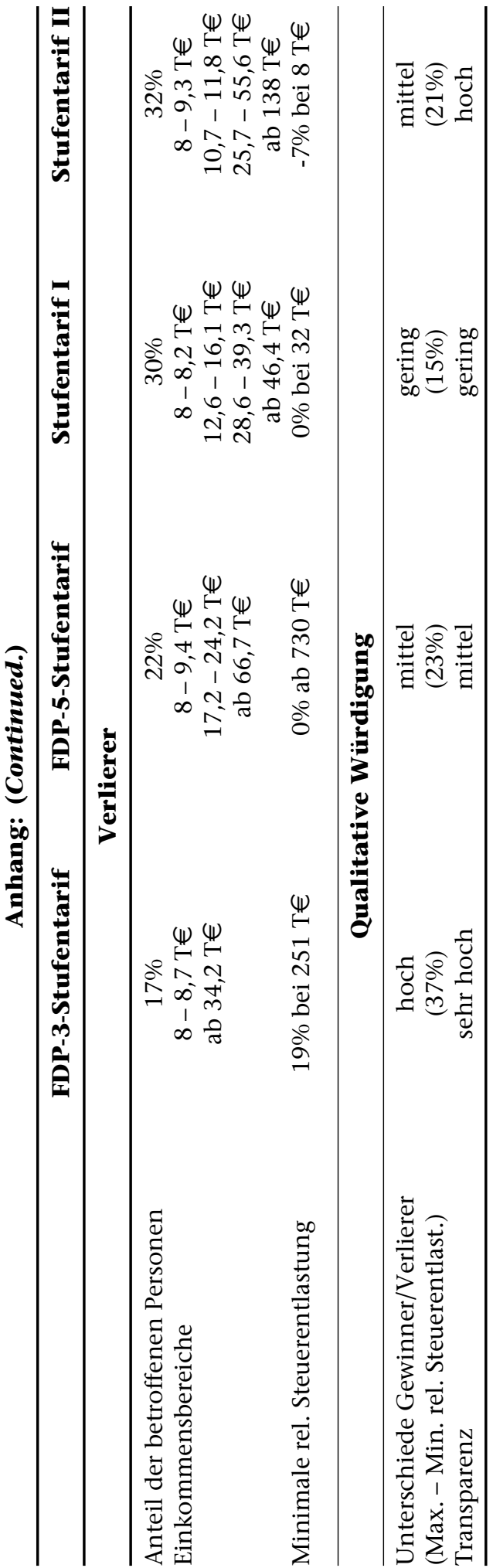

